\title{
New Approach of Dipterocarpus alatus 0il as a Permeation Enhancer in Ibuprofen Gel
}

\author{
Supawadee Daodee*, Orawan Monthakantirat, Yaowared Chulikhit \\ Division of Pharmaceutical Chemistry, Faculty of Pharmaceutical Sciences, Khon Kaen University, THAILAND.
}

\begin{abstract}
Background: Yang-na tree has a scientific name as Dipterocapus alatus Roxb. Ex G.Don in family Dipterocarpaceae. Many parts of this tree can be used especially for $D$. alatus oil. Objectives: To develop basic formula of ibuprofen gel composed of $D$. alatus oil as a permeation enhancement agent from natural source. Materials and Methods: This study composed of formulation development, physico-chemical property testing, permeation study and stability study. Results: The suitable ibuprofen gel formulation consisted of 0.5 percent $D$. alatus oil for carbopol and 1 percent $D$. alatus oil for carboxymethylcellulose as gelling agent when evaluated by side-by-side diffusion cell and physico-chemical properties. Conclusion: This study could get the basic ibuprofen gel formula containing $D$. alatus oil as the permeation enhancer which gave the value added of this oil to apply and use in pharmaceutical.

Key words: Dipterocapus alatus, Ibuprofen, Permeation enhancer, Carbopol, Carboxymethylcellulose, Gel.
\end{abstract}

\section{INTRODUCTION}

Dipterocarpus alatus is one kind of plant in family Dipterocarpaceae which plays a major role in the economy in many Asian countries. This plant can grow naturally in tropical forest of Southeast Asia including Thailand which can be used as an important plant for reforestation planning programs. ${ }^{1}$ Production of oil from $D$. alatus is very high and can find in many parts of Thailand and is one of the potential natural oil which might be used for drug formulation. The previous study from some scientists revealed that many kinds of chemical compound such as terpenes are found in natural oils and reviewed the use of different natural products including essential oils as potential permeation enhancer for transdermal drug delivery system. ${ }^{2}$ Numerous terpenes have long been used as medicines as well as flavoring and fragrance agents and can be used as skin permeation enhancers. ${ }^{3-6}$

Nowadays, NSAIDs (Nonsteroidal antiinflammatory drugs) is almost the common formulation to treat many kind of symptoms. NSAIDs are used primarily to treat inflammation, mild to moderate pain, and fever. Specific uses include the treatment of headache, arthritis, ankylosing spondylitis, sports injuries, and menstrual cramps. ${ }^{7,8}$ Ibuprofen belongs to a class of NSAIDs and is one of the most common NSAIDs. Ibuprofen is used for the treatment of mild to moderate pain, inflammation and fever caused by many and diverse diseases. It is used for treating menstrual cramps (dysmenorrhea), osteoarthritis, rheumatoid arthritis, and juvenile idiopathic arthritis. Ibuprofen formulations commercially in the market are in oral and topical forms. Ibuprofen may cause ulceration of the stomach or intestine, and the ulcers may bleed. So, the other type of drug administration was developed and used locally to avoid its side effect. Topical preparation prevents the metabolism of drug in the liver, avoids gastrointestinal disorders and the risk of inconveniences of intravenous therapy. Topical ibuprofen gel formulation is a good choice of using. However the skin might be the barrier
Submission Date: 03-06-2021; Revision Date: 19-08-2021; Accepted Date: 09-11-2021.

DOI: 10.5530/ijper.56.1.4 Correspondence: Dr. Supawadee Daodee Associate Professor, Division of Pharmaceutical Chemistry, Faculty of Pharmaceutical

Sciences, Khon Kaen University, Khon Kaen, THAILAND.

E-mail: csupawad@kku.ac.th

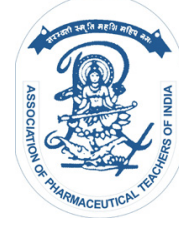

www.ijper.org 
resistance for the drug to permeate through that rough area. This problem should be concerned to improve the effective of treatment. Permeation enhancer is one of choice to resolve and might me added into the formulation to help ibuprofen through the skin membrane for treatment which is one long-standing approach for improving transdermal drug delivery system. ${ }^{10-12}$ Permeation enhancer is also called sorption promoter or accelerant. Numerous compounds have been used and evaluated for penetration enhancing activity, including sulphoxides such as dimethylsulphoxide, azones such as laurocapram, pyrrolidones e.g. 2-pyrrolidone, alcohols and alkanols (ethanol, or decanol), glycols (e.g. propylene glycol) and terpenes. ${ }^{13-15}$ Many modes of actions have been identified for skin penetration enhancers such as the intercellular lipid matrix in which the accelerants may disrupt the packing skin membrane layer, the intracellular keratin or through increasing drug partitioning into the tissue by acting as a solvent for the permeant within the skin membrane. The other potential actions may be come from altering metabolic activity within the skin, or influence on the thermodynamic activity or solubility of the drug. ${ }^{16}$ At the present time, some of the natural essential oil can be used as the permeation enhancer as the essentials oils show a large range of chemically acceptable and relatively safe penetration enhancers to help percutaneous drug absorption and are considered as safe compounds for medicinal purpose with no skin toxicity, but only mild irritation. ${ }^{17-20} \mathrm{D}$. alatus oil was formerly studied about the property of permeation enhancement for some drug and chemical substances and there was some study which applied to use D. alatus oil in the ketoconazole shampoo formula and reported the value of this natural oil in the drug formula., ${ }^{21,22}$

Thus, the aim of this study was to develop the model ibuprofen gel formulation composed with $D$. alatus oil as the permeation enhancer and to evaluate the physicochemical properties of the formulation.

\section{MATERIALS AND METHODS}

\section{Materials and equipments}

Side-by-side diffusion cell was used in permeation study. UV-Visible spectrophotometer, viscometer and differential scanning calorimeter were product of Shimadzu ${ }^{\circledR}$, Brookfield ${ }^{\circledR}$ and Mettler Toledo ${ }^{\circledR}$ as followed. Ibuprofen was purchased from SigmaAldrich $^{\circledR}$. Sodium dihydrogenphosphate, disodium hydrogenphosphate, dimethyl formamide, sodium hydroxide, triethanolamine, ethanol and octanol were purchased from Merck ${ }^{\circledR}$. Carbopol 940 and propylene glycol were of BP grade. Carboxymethylcellulose was purchased from Fluka ${ }^{\circledR}$. D. alatus oil was kindly supported from Yangna research cluster. Porcine abdominal membrane was purchased from the fresh market in Khon Kaen province.

\section{Preparation of drug solutions}

The stock standard solutions $(1 \mathrm{mg} / \mathrm{ml})$ of ibuprofen was prepared and dissolved in methanol and then diluted with phosphate buffer to the appropriate concentration for permeation studies. All solutions were freshly prepared daily.

\section{Preparation of standard ibuprofen solution}

The stock solution of ibuprofen $(1 \mathrm{mg} / \mathrm{ml})$ was diluted to the appropriate concentration $(0.5-30 \mu \mathrm{g} / \mathrm{ml})$ with $50 \%$ methanol and then measured the absorbance at $222 \mathrm{~nm}$. The standard curve was plotted between absorbance and concentration.

\section{In vitro drug permeation study}

The permeation study of ibuprofen gel was carried out by side-by-side diffusion cell which the temperature was controlled at $37 \pm 0.5^{\circ} \mathrm{C}$ in water bath. Porcine abdominal membrane was first immersed in $D$. alatus oil for $30 \mathrm{~min}$ while the control was immersed in phosphate buffer solution and 5\%dimethyl formamide. The membrane was clamped in side-by-side diffusion cell between donor and receptor compartment. Three $\mathrm{ml}$ of phosphate buffer solution at $\mathrm{pH} 7.4$ was added into receptor compartment. The donor compartment was added with appropriate amount of ibuprofen gel in phosphate buffer at $\mathrm{pH}$ 7.4. Both compartments were magnetically stirred throughout the experiment at $600 \mathrm{rpm}$. The solution in receptor compartment was sampling at various time intervals and immediately refilled with the same volume $(1 \mathrm{ml})$ of fresh buffer solutions. Sample solutions were analyzed for the permeated content by UV-Vis spectrophotometry. The absorbance of resulting solutions was measured at its maximum wavelength which was $222 \mathrm{~nm}$. All the formulations were also tested for their permeation ability.

\section{Preparation of model ibuprofen gel composed with $D$. alatus oil}

All excipients including ibuprofen, D. alatus oil, gelling agent and other excipients which depend on the type of gelling agent were prepared as described below. The resulting ibuprofen formulations with the different type of gellling agent and variation percentage of $D$. alatus oil were also prepared to get the appropriate gel 
formulation. Carbopol 940 and carboxymethylcellulose were used as the gelling agents in this study.

When carbopol 940 was used (formulation A1-A11), the formulation would contain ibuprofen, D. alatus oil, propylene glycol, triethanolamine and ultrapure water as listed in Table 1. Ibuprofen powder was dissolved by ethanol and proper to add into the polymer. The proper amount of polymer was dispersed in ultrapure water in a beaker and stirred using a magnetic stirrer. Propylene glycol was added and stirred until a homogeneous gel was achieved. The adequate amount of triethanolamine was added to neutralize free carboxylic acid groups of carbopol 940 to the desired $\mathrm{pH}$. D. alatus oil was added to the previous polymer dispersion with slightly stirring. Another gelling agent, carboxymethylcellulose (formulation B1-B9), the formulation contained ibuprofen, D. alatus oil, propylene glycol and ultrapure water (Table 1). The formulation was prepared as described above but adding triethanolamine is not necessary. Gel formulation was stand for $30 \mathrm{~min}$ before adding $D$. alatus oil and ibuprofen. The compositions of all developed formula were shown in Table 1.

\section{Evaluation of ibuprofen gel composed of $D$. alatus oil}

\section{Appearance}

All gel formulations were examined by visual estimation for their color, clarity, odor, homogeneity and phase separation.

\section{Evaluation of $\mathrm{pH}$}

The $\mathrm{pH}$ of each gel formulation was measured using $\mathrm{pH}$ meter. The results were shown as mean from three replications.

\section{Spreadability}

This test was determined using the method and apparatus which modified in-house. Glass plate was used and fixed on the table. Gel $(0.5 \mathrm{~g})$ was weighed and placed on the center of glass plate. Another glass plate (with $500 \mathrm{~g}$ of material) was placed on the gel like a sandwich for 5 min. The diameter of spreaded gel was measured in

\section{Table 1: The composition in ibuprofen gel formulation composed with $D$. alatus oil using carbopol 940 and} carboxymethylcellulose as gel forming agent.

\begin{tabular}{|c|c|c|c|c|c|c|c|c|c|}
\hline $\begin{array}{c}\text { Ingredients } \\
(\%)\end{array}$ & Ibuprofen & $\begin{array}{c}\text { D. alatus } \\
\text { oil }\end{array}$ & Carbopol940 & CMC & Ethanol & 2-propanol & $\begin{array}{c}\text { Propylene } \\
\text { glycol }\end{array}$ & Triethanolamine & $\begin{array}{c}\text { Ultrapure } \\
\text { Water }\end{array}$ \\
\hline \multicolumn{10}{|c|}{ Carbopol (A) } \\
\hline A1 & 5 & 1 & 1 & - & 0 & 0 & 10 & 1 & qs to 100 \\
\hline A2 & 5 & 2 & 1 & - & 0 & 0 & 10 & 1 & qs to 100 \\
\hline A3 & 5 & 1 & 1 & - & 0 & 0 & 10 & 10 drops & qs to 100 \\
\hline A4 & 5 & 1 & 1 & - & 5 & 0 & 10 & 10 drops & qs to 100 \\
\hline A5 & 5 & 1 & 1 & - & 5 & 0 & 10 & 6 drops & qs to 100 \\
\hline A6 & 5 & 1 & 1 & - & 5 & 0 & 20 & 4 drops & qs to 100 \\
\hline A7 & 5 & 1 & 1 & - & 5 & 0 & 20 & 2 drops & qs to 100 \\
\hline A8 & 5 & 1 & 1 & - & 10 & 0 & 20 & 2 drops & qs to 100 \\
\hline A9 & 2.5 & 1 & 1 & - & 10 & 0 & 20 & 2 drops & qs to 100 \\
\hline A10 & 5 & 1 & 1 & - & 15 & 0 & 20 & 2 drops & qs to 100 \\
\hline A10.1 & 5 & 1 & 1 & - & 15 & 0 & 20 & 2 drops & qs to 100 \\
\hline A10.2 & 5 & 2 & 1 & - & 15 & 0 & 20 & 2 drops & qs to 100 \\
\hline A10.3 & 5 & 3 & 1 & - & 15 & 0 & 20 & 2 drops & qs to 100 \\
\hline A10.4 & 5 & 0.5 & 1 & - & 15 & 0 & 20 & 2 drops & qs to 100 \\
\hline A11 & 5 & 0 & 1 & - & 15 & 0 & 20 & 2 drops & qs to 100 \\
\hline \multicolumn{10}{|c|}{ Carboxymethylcellulose, CMC (B) } \\
\hline B1 & 5 & 1 & - & 1 & 0 & 0 & 10 & 0 & qs to 100 \\
\hline B2 & 5 & 1 & - & 1 & 15 & 0 & 20 & 0 & qs to 100 \\
\hline B3 & 5 & 1 & - & 1 & 0 & 15 & 20 & 0 & qs to 100 \\
\hline B4 & 5 & 1 & - & 2 & 0 & 15 & 20 & 0 & qs to 100 \\
\hline B5 & 5 & 1 & - & 2 & 15 & 0 & 20 & 0 & qs to 100 \\
\hline B6 & 5 & 1 & - & 3 & 15 & 0 & 20 & 0 & qs to 100 \\
\hline B7 & 5 & 0.5 & - & 3 & 15 & 0 & 20 & 0 & qs to 100 \\
\hline B8 & 5 & 2 & - & 3 & 15 & 0 & 20 & 0 & qs to 100 \\
\hline B9 & 5 & 0 & - & 3 & 15 & 0 & 20 & 0 & qs to 100 \\
\hline
\end{tabular}


three replications and then percent spreadability was calculated as \%spread by area.

\section{Viscosity measurement}

The measurement of viscosity of all gel formulations was done with a Brookfield viscometer. The viscometer was set to rotate from $0-100 \mathrm{r} / \mathrm{min}$ using T-bar spindle no.96. At each speed, the viscosity was measured and recorded.

\section{Thermal property testing}

Differential scanning calorimeter (DSC) was used for the study of thermal property in gel formulation. Gels $(9-10 \mathrm{~g})$ were put into the sample pan of DSC. The reference pan was used as a control in the experiment. The temperature was set start from 30 to $300^{\circ} \mathrm{C}$ with $10^{\circ} \mathrm{C}$ incrementally. DSC thermogram for each gel formulation was plotted and recorded.

\section{Drug content study}

Half gram of gel was weighed and sonicated in methanol for $5 \mathrm{~min}$ when used carbopol 940 and $30 \mathrm{~min}$ for carboxymethylcellulose. The sample was then filtered with whatman no. 1 and adjusted to $10 \mathrm{ml}$ with methanol in volumetric flask. One $\mathrm{ml}$ of the sample solution was pipetted into $50 \mathrm{ml}$ volumetric flask, adjusted to volume with $50 \%$ methanol and then measured the absorbance at wavelength $222 \mathrm{~nm}$. Methanol (50\%) was used as a blank in the study. Determinations were made in triplicate.

\section{Preliminary stability study}

The stability of gel formulation was evaluated in two conditions, room temperature and 45 degree centigrade over a period of six weeks. Ibuprofen content was determined periodically after gel preparation at $0,2,4$ and 6 weeks. The method for determination was the same as described above for drug content study.

\section{RESULTS}

\section{Physico-chemical characteristic of ibuprofen gel formulation}

From visual estimation, the results of all developed formulations were shown in Table 2. Some of the gel

Table 2: Characteristic and pH of ibuprofen gel formulation using carbopol 940 and carboxymethylcellulose as gelling agent.

\begin{tabular}{|c|c|c|c|c|}
\hline Formulation code & Characteristic & Color & Odor & Average $\mathrm{pH} \pm \mathrm{SD}$ \\
\hline A1 & Moderate sticky mass, not smooth & Yellow-white & normal & $6.43 \pm 0.01$ \\
\hline A2 & Moderate sticky mass, not smooth & yellow & normal & $6.43 \pm 0.01$ \\
\hline A3 & Moderate sticky mass, not smooth & White-yellow & normal & $7.00 \pm 0.00$ \\
\hline A4 & Moderate sticky mass, not smooth & Yellow-white & normal & $6.20 \pm 0.01$ \\
\hline A5 & Moderate sticky mass, not smooth & Yellow-white & normal & $5.70 \pm 0.02$ \\
\hline A6 & Slightly sticky mass, smooth & yellow & normal & $5.72 \pm 0.01$ \\
\hline A7 & Slightly sticky mass, not smooth & yellow & normal & $5.35 \pm 0.01$ \\
\hline A8 & Slightly sticky mass, slightly smooth & yellow & normal & $5.43 \pm 0.02$ \\
\hline A9 & Slightly smooth & yellow & normal & $5.46 \pm 0.01$ \\
\hline A10 & Smooth & White-yellow & normal & $5.41 \pm 0.01$ \\
\hline A10.1 & Smooth & White-yellow & normal & $5.54 \pm 0.02$ \\
\hline A10.2 & Smooth & Dark yellow & normal & $5.54 \pm 0.01$ \\
\hline A10.3 & Slightly Smooth & Dark yellow & normal & $5.67 \pm 0.00$ \\
\hline A10.4 & Smooth & Yellow-white & normal & $5.46 \pm 0.01$ \\
\hline A11 & Smooth & White & normal & $5.72 \pm 0.02$ \\
\hline B1 & Separate layer & Clear solution on top layer & normal & $6.90 \pm 0.00$ \\
\hline B2 & Separate layer & Clear solution on top layer & normal & $6.93 \pm 0.01$ \\
\hline B3 & Separate layer & Clear solution on top layer & Strong odor & $6.88 \pm 0.00$ \\
\hline B4 & Slightly smooth & yellow & Strong odor & $7.30 \pm 0.01$ \\
\hline B5 & Slightly smooth & yellow & normal & $7.44 \pm 0.00$ \\
\hline B6 & Smooth & yellow & normal & $7.29 \pm 0.02$ \\
\hline B7 & Smooth & Yellow-white & normal & $7.18 \pm 0.00$ \\
\hline B8 & Smooth & Dark yellow & normal & $7.17 \pm 0.00$ \\
\hline B9 & Smooth & White & normal & $6.69 \pm 0.01$ \\
\hline
\end{tabular}


formulation showed smooth character, no grittiness and good homogeneity as in formulation A9-A11 and B6-B9. The $\mathrm{pH}$ of gel was ranged between $5.35-7.00$ for A1-A11 and 6.88-7.44 for B1-B9 as listed in Table 2. The most appropriate gel formulation estimated from physico-chemical properties without $D$. alatus oil was A11 from carbopol 940 and B9 for carboxymethylcellulose. These two formulations were used as the basic formulation then modified by varying percentage of $D$. alatus oil to be formulation code A10.1, 10.2, 10.4 and B6, 7, 8. For A10.3 which was the formulation containing $3 \%$ D. alatus oil, the phase separation was observed, then we stopped the study for this formulation. It might be due to very high content of oil phase in gel formulation affect the homogeneity of formulation.

Viscosity of gel formulation was evaluated in A10.1, A10.2 and A10.4 as shown in Figure 1. It was found that the highest viscosity formulation was achieved from A10.1 and B6. Moreover, the formulation with carboxymethylcellulose as gelling agent had strongly higher viscosity than the formulation using carbopol 940. Hence, the viscosity from the formulation containing carboxymethylcellulose in this study could not be measured and no viscosity profile was recorded. When using carbopol as gelling agent, the solution would have a low $\mathrm{pH}$. Some neutralizing agent as triethanolamine was added to increase the $\mathrm{pH}$ and caused the dispersion of gel. The selection between carbopol and CMC depended on many factors such as the compatibility of active compound with other excipients in the formulation especially for the solubility.

The spreadability of model gel formulation was considered high by having a low spread of time. The efficacy of the gel formulation depended on their spread which gel spreading could help the consistence and uniform application of gel to skin. Good spreadability of gel formulation was considered an important factor in patient compliance. From the result of this study as shown in Table 3, highest \% spreadability was found

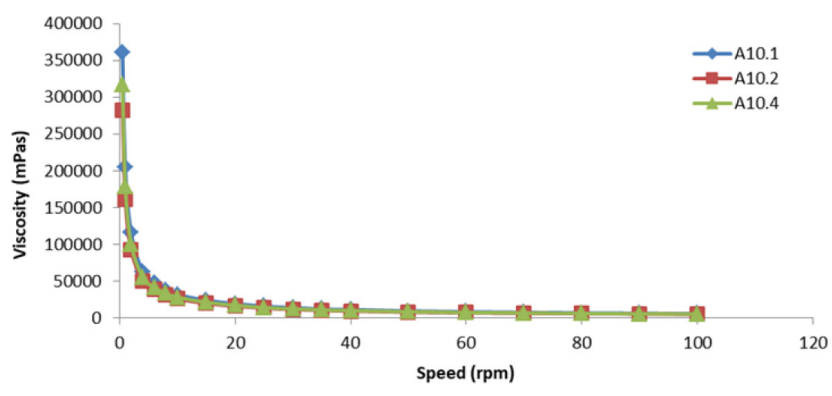

Figure 1: Viscosity profile of ibuprofen gel formulation from variation content of $D$. alatus oil and carbopol 940 was used as gelling agent.

\begin{tabular}{|c|c|}
\hline \multicolumn{2}{|c|}{ Table 3: Spreadability of ibuprofen gel formulations } \\
\hline Formulations & Percent spread by area \pm SD \\
\hline A10.1 & $375 \pm 13.6$ \\
\hline A10.2 & $396 \pm 8.6$ \\
\hline A10.3 & $380 \pm 13.4$ \\
\hline A10.4 & $382 \pm 8.1$ \\
\hline A11 & $258 \pm 6.0$ \\
\hline B6 & $250 \pm 5.7$ \\
\hline B7 & $253 \pm 7.6$ \\
\hline B8 & $250 \pm 2.6$ \\
\hline B9 & $250 \pm 7.0$ \\
\hline
\end{tabular}
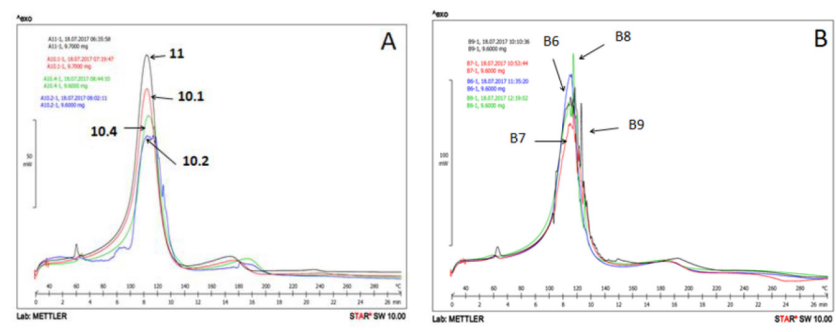

Figure 2: Differential Scanning Calorimetry (DSC) thermograms of ibuprofen gel formulations from two different gelling agent and variation content of $D$. alatus oil in the formulation. (A: carbopol 940, B: carboxymethylcellulose).

in A10.2 for carbopol 940 as gelling agent and B6 for carboxymethylcellulose. Moreover, higher spreadability between these two gelling agents was found in carbopol 940. Gel formulation containing Carbopol 940 provided better character.

The thermal property of gel formulation was shown as DSC thermogram in Figure 2. The similar pattern was found between each formulation including the formulation from two gelling agents. These thermograms revealed the homogeneity of the composition in all formulations in this study. Melting point of gel formulation was range between $100-130^{\circ} \mathrm{C}$.

\section{Permeabilty of ibuprofen gel formulation composed with $D$. alatus oil}

From the result of the permeability study, Formulation containing with $0.5 \%$ D. alatus oil showed highest permeation than the other formulations in the group of using carbopol 940 as gelling agent. While the formulation using carboxymethylcellulose, the appropriate $D$. alatus content which performed highest permeation was $1 \%$. The permeation profiles were shown in Figure 3. The result from this permeation study was not related to the linear relationship between percentage of $D$. alatus oil and permeability of ibuprofen gel. It might be caused from the higher content of $D$. alatus oil obstructed the 

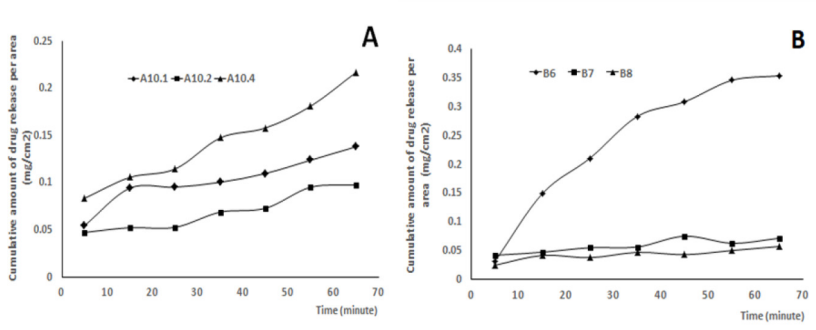

Figure 3: Permeation profile of ibuprofen gel formulation using different gelling agent and variation of $D$.alatus oil in formulation (A: carbopol 940, B: carboxymethylcellulose).

channel of permeation at the membrane which could observed from the surface of the skin after finish the experiment. Thus, higher $D$. alatus oil content did not mean better permeation of ibuprofen gel formulation.

\section{Preliminary stability study of ibuprofen gel formulation}

From the stability study, it was found that there was no significance statistical different of ibuprofen content in the formulation during the collecting time up to 6 weeks at room temperature condition. It was controversy to the stability at $45^{\circ} \mathrm{C}$ conditioned in which the content increased when the collecting time up. It might be come from low sensitivity of the analytical method, UV spectrophotometry. During the collecting time increases, gel formulation turned from light yellow to dark yellow which might interfere to absorbance measurement. Thus, the further study should concern to the better analytical method when the stability was conducted.

\section{DISCUSSION}

The new approach for using $D$. alatus oil as a natural permeation enhancer in gel formulation was revealed in this study. The study conformed to the previous study which $D$. alatus oil could be used to improve the permeation of some model compounds such as ibuprofen, ketoconazole and caffeine through newborn pork skin membrane. ${ }^{21}$ Moreover, D. alatus oil was formerly study in one shampoo formulation containing ketoconazole and trended to increase the permeation of active compound in the formulation. ${ }^{22}$ Extended study was performed using this oil as an enhancer in gel formulation which composed of ibuprofen as an active ingredient. Some natural oil was also used in the same manner as permeation enhancer as reported by Aggarwal and co-workers in 2012, ${ }^{17}$ for investigating the development of olanzapine transdermal delivery system using natural oils as permeation enhancers. Corn (maize) oil, groundnut oil and jojoba oil on in vitro permeation of olanzapine across rat skin were studied. The other similar study was revealed using some essential oils to enhance the permeation of 5-fluorouracil through excised human skin. ${ }^{20}$ Eucalyptus oil and chenopodium oil were found to be very effective (30-fold increase), while Ylang ylang oil was mildly effective (8-fold increase) and anise oil had little activity (3-fold increase). Some former study revealed some natural oils such as clove oil and angelica oil for the penetration enhancer of ibuprofen for oral therapy which could disturb the highly ordered intercellular lipid structure between corneocytes in the subcutaneous layer and could increase the bioavailability of drug substance. ${ }^{23}$ Moreover, coconut oil and pistachio oil were also enhanced the permeation of clotrimazole gel through rat skin by the study of Fox and Gerber in 2011. ${ }^{24}$

This study only investigated the new approach of natural oil, D. alatus oil in a model ibuprofen gel formulation and varying type of gelling agent and its content was also evaluated. The penetration enhancer might function by altering the barrier characteristics of skin membranes or by increasing the drug solubility inside the skin hence enhancing the partition coefficient of drug through the skin. However, limitation of this study was occurred from using only the composition of basically gel formula. The other excipients in gel formulation to improve the usability and stability of the product such as some coloring, smelling agent or antioxidant did not added in this study. The further study should rearrange and vary some of the other excipient in the formulation to get the better appropriate ibuprofen gel formulation and should applied to clinically study and compared the efficacy of ibuprofen gel formulation with and without D. alatus oil.

\section{CONCLUSION}

From this study, the model ibuprofen gel formulation composed of $D$. alatus oil was developed and evaluated for the physico-chemical property, permeability and stability. From all testing and evaluating, the appropriate gelling agent was carbopol 940 which could give the better property compared to carboxymethylcellulose. Moreover, $D$. alatus oil could be a permeation enhancer in model ibuprofen gel formulation which could increase the efficacy of treatment. The results from this study revealed the efficacy and usefulness of $D$. alatus oil and should further apply to the other type of drug and use as the permeation enhancer in the other formulation. D. alatus oil, new permeation enhancer from natural origin trend to become popular as it offers several 
benefits over the synthetic substances such as sustainable product from the tree of Royal Initiative project, lower cost and eco domestic product.

\section{ACKNOWLEDGEMENT}

The authors acknowledge the financial support received from Khon Kaen University under Research and Technology Transfer Affairs (KKU-YN010) and the material, Dipterocarpus alatus oil from Yangna research cluster.

\section{CONFLICT OF INTEREST}

The authors declare no conflict of interest.

\section{ABBREVIATIONS}

UV: Ultraviolet; D. alatus: Dipterocarpus alatus; DSC: Differential Scanning Calorimeter.

\section{REFERENCES}

1. Dang-Le AT, Edelin C, Le-Cong K. Ontogenetic variations in leaf morphology of the tropical rain forest species Dipterocarpus alatus Roxb. ex G. Don. Trees. 2013;27(3):773-86. doi: 10.1007/s00468-012-0832-2 ex G.

2. Jain AK, Thomas NS, Panchagnula R. Transdermal drug delivery of imipramine hydrochloride. I. Effect of terpenes. J Control Release. 2002;79(1-3):93-101. doi: 10.1016/s0168-3659(01)00524-7, PMID 11853921.

3. Cornwell PA, Barry BW, Bouwstra JA, Gooris GS. Modes of action of terpene penetration enhancers in human skin; Differential scanning calorimetry, smallangle X-ray diffraction and enhancer uptake studies. International Journal of Pharmaceutics. 1996;127(1):9-26. doi: 10.1016/0378-5173(95)04108-7.

4. Cornwell PA, Barry BW. Sesquiterpene components of volatile oils as skin penetration enhancers for the hydrophilic permeant 5-fluorouracil. J Pharm Pharmacol. 1994;46(4):261-9. doi: 10.1111/j.2042-7158.1994.tb03791.x, PMID 8051608.

5. Vaddi HK, Ho PC, Chan SY. Terpenes in propylene glycol as skin-penetration enhancers: permeation and partition of haloperidol, Fourier transform infrared spectroscopy, and differential scanning calorimetry. J Pharm Sci. 2002;91(7):1639-51. doi: 10.1002/jps.10160, PMID 12115825.

6. Vaddi HK, Wang LZ, Ho PC, Chan SY. Effect of some enhancers on the permeation of haloperidol through rat skin in vitro. Int $\mathrm{J}$ Pharm. 2001;212(2):247-55. doi: 10.1016/s0378-5173(00)00616-5, PMID 11165082.

7. Simon LS. Biologic effects of non-steroidal anti-inflammatory drugs. Curr Opin Rheumatol. 1997;9(3):178-82. doi: 10.1097/00002281-199705000-00002, PMID 9204251

8. Laine L. Approaches to non-steroidal anti-inflammatory drug use in the high-risk patient. Gastroenterology. 2001;120(3):594-606. doi: 10.1053/ gast.2001.21907, PMID 11179238.
9. Britannica. The editors of encyclopaedia; Jun 24 2021. Ibuprofen. Encyclopaedia Britannica. Available from: https://www.britannica.com/ science/ibuprofen [cited 25/11/2021].

10. Wang FL, Ji HM, Zhu JY, Xu GJ, Guan YZ, Chen YJ. Penetration enhancement effect of turpentine oil on transdermal film of ketorolac. Trop J Pharm Res. 2015;14(8):1341-8. doi: 10.4314/tjpr.v14i8.4.

11. Naik A, Kalia YN, Guy RH. Transdermal drug delivery: Overcoming the skin's barrier function. Pharm Sci Technol Today. 2000;3(9):318-26. doi: 10.1016/ s1461-5347(00)00295-9, PMID 10996573.

12. Narishetty ST, Panchagnula R. Transdermal delivery of zidovudine: effect of terpenes and their mechanism of action. J Control Release. 2004;95(3):367-79. doi: 10.1016/j.jconrel.2003.11.022, PMID 15023449.

13. Patil UK, Saraogi R. Natural products as potential drug permeation enhancer in transdermal drug delivery system. Arch Dermatol Res. 2014;306(5):419-26. doi: 10.1007/s00403-014-1445-y, PMID 24481830.

14. Chen Y, Quan P, Liu X, Wang M, Fang L. Novel chemical permeation enhancers for transdermal drug delivery. Asian J Pharm Sci. 2014;9(2):51-64. doi: 10.1016/j.ajps.2014.01.001.

15. Pathan IB, Setty CM. Chemical penetration enhancers for transdermal drug delivery systems. Trop J Pharm Res. 2013;8(2):173-9. doi: 10.4314/tjpr. v8i2.44527.

16. Alexander A, Dwivedi S, Ajazuddin GTK, Giri TK, Saraf S, Saraf S, et al. Approaches for breaking the barriers of drug permeation through transdermal drug delivery. J Control Release. 2012;164(1):26-40. doi: 10.1016/j. jconrel.2012.09.017, PMID 23064010.

17. Aggarwal G, Dhawan S, HariKumar SL. Natural oils as skin permeation enhancers for transdermal delivery of olanzapine: In vitro and in vivo evaluation. Curr Drug Deliv. 2012;9(2):172-81. doi: 10.2174/156720112800234567, PMID 22023211.

18. Aqil M, Ahad A, Sultana $Y$, Ali A. Status of terpenes as skin penetration enhancers. Drug Discov Today. 2007;12(23-24):1061-67. doi: 10.1016/j. drudis.2007.09.001, PMID 18061886.

19. Vashisth I, Ahad A, Aqil M, Agarwal SP. Investigating the potential of essential oils as penetration enhancer for transdermal losartan delivery: Effectiveness and mechanism of action. Asian J Pharm Sci. 2014;9(5):260-7. doi: 10.1016/j. ajps.2014.06.007.

20. Williams AC, Barry BW. Essential oils as novel human skin penetration enhancers. International Journal of Pharmaceutics. 1989;57(2):R7-9. doi: 10.1016/0378-5173(89)90310-4.

21. Daodee S, Punya T, Chansri N. Application of Dipterocarpus alatus oil for Permeation Enhancement of Some compounds. Res J Pharm Technol. 2018;11(1):245-8. doi: 10.5958/0974-360X.2018.00045.8.

22. Daodee S, Monthakantirat O, Chulikhit $\mathrm{Y}$, Punya T. Development of ketoconazole with Dipterocarpus alatus oil as a permeation enhancer. Indian J Pharm Educ Res. 2018;52(4s2): S197-201.

23. Chen J, Jiang QD, Wu YM, Liu P, Yao JH, Lu Q, et al. Potential of essential oils as penetration enhancers for transdermal administration of ibuprofen to treat dysmenorrhoea. Molecules. 2015;20(10):18219-36. doi: 10.3390/ molecules201018219, PMID 26457698.

24. Fox LT, Gerber M, Plessis JD, Hamman JH. Transdermal drug delivery enhancement by compounds of natural origin. Molecules. 2011;16(12):10507-40. doi: 10.3390/molecules161210507. 
PICTORIAL ABSTRACT

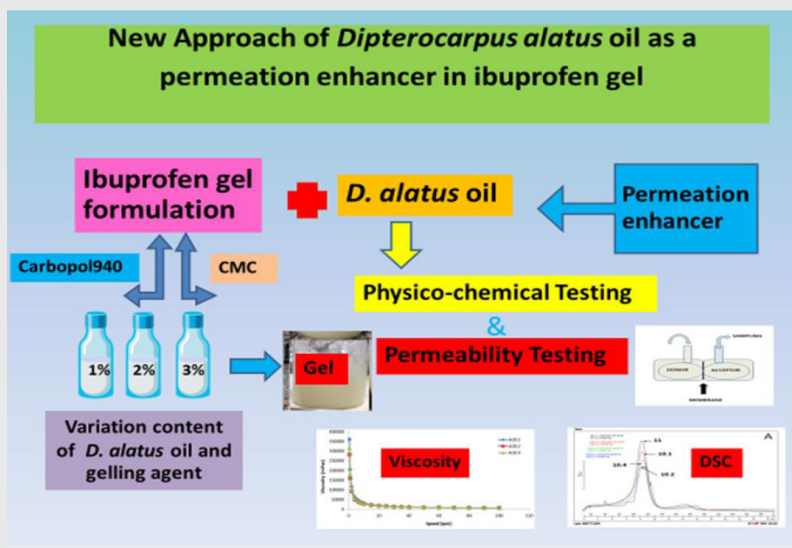

\section{SUMMARY}

- The model of ibuprofen gel formulation with $D$. alatus oil as permeation enhancer was proposed and developed in this study using carbopol 940 and carboxymethylcellulose as gelling agent.

- The developed formulation was acceptable for the general basic requirement of desired specification for visual estimation, $\mathrm{pH}$, thermal property and viscosity.

- New approach and concept for developing the model formulation using $D$. alatus oil as a permeation enhancer should be further studied to the other topical formulation.

\section{About Authors}

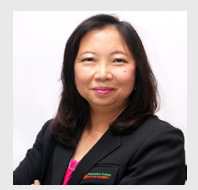

Supawadee Daodee, Working as Associate Professor in Division of Pharmaceutical Chemistry, Faculty of Pharmaceutical Sciences, Khon Kaen University. She has wide knowledge and experience in the field of pharmaceutical analysis, pharmaceutics, pharmaceutical chemistry and quality control of herbal product. She is currently focusing on Thai traditional medicine and quality control.

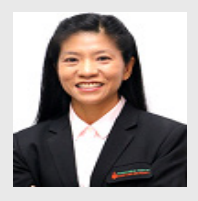

Orawan Monthakantirat, Working as Assistant Professor in Division of Pharmaceutical Chemistry, Faculty of Pharmaceutical Sciences, Khon Kaen University. She has wide knowledge and experience in the field of phytochemical analysis, pharmaceutical chemistry and biological activity testing.

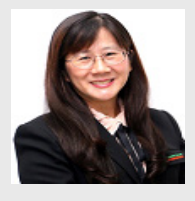

Orawan Monthakantirat, Working as Assistant Professor in Division of Pharmaceutical Chemistry, Faculty of Pharmaceutical Sciences, Khon Kaen University. She has wide knowledge and experience in the field of Phytochemical analysis, Pharmaceutical chemistry and Biological activity testing.

Cite this article: Daodee S, Monthakantirat O, Chulikhit Y. New Approach of Dipterocarpus alatus Oil as a Permeation Enhancer in Ibuprofen Gel. Indian J of Pharmaceutical Education and Research. 2022;56(1):24-31. 